\title{
Generating Totally Positive Toeplitz Matrix from an Upper Bidiagonal Matrix
}

\author{
Mohamed A. Ramadan1, Mahmoud M. Abu Murad² \\ ${ }^{1}$ Department of Mathematics, Faculty of Science, Menoufia University, Al Minufya, Egypt \\ ${ }^{2}$ Department of Mathematics, Faculty of Science, Zagazig University, Ash Sharqiyah, Egypt \\ Email: ramadanmohamed13@yahoo.com,mramadan@eun.eg, abomorad1978@yahoo.com
}

Received 17 September 2015; accepted 29 November 2015; published 2 December 2015

Copyright (C) 2015 by authors and Scientific Research Publishing Inc.

This work is licensed under the Creative Commons Attribution International License (CC BY).

http://creativecommons.org/licenses/by/4.0/

(c) (i) Open Access

\begin{abstract}
In this paper, we construct one of the forms of totally positive Toeplitz matrices from upper or lower bidiagonal totally nonnegative matrix. In addition, some properties related to this matrix involving its factorization are presented.
\end{abstract}

\section{Keywords}

Totally Positive Matrix, Totally Nonnegative Matrix, Toeplitz Matrix, $L U$ Factorization

\section{Introduction}

Total positive matrices arise in many areas in mathematics, and there has been considerable interest lately in the study of these matrices. For background information see the most important survey in this field by T. Ando [1]. See also [2].

A matrix $A$ is said to be totally positive, if every square submatrix has positive minors and $A$ is said to be totally nonnegative, and if every square submatrix has nonnegative minors. While it is well known that many of the nontrivial examples of totally positive matrices are obtained by restricting certain kernels to appropriate finite subsets of $R$ (see, for example, Ando ([1], p. 212) or Pinkus ([3], p. 2). For Toeplitz matrices, that is, matrices of the form $T=\left(t_{i-j}\right)_{i, j=1}^{n}$, a complete characterization of the total positivity, in terms of certain entire functions, has been studied in a series of references by Ando [1], Pinkus [3] and S.M. Fallat, C.R. Johnson [4].

Expressing a matrix as a product of lower triangle matrix $L$ and an upper triangle matrix $U$ is called a $L U$ factorization. Such factorization is typically obtained by reducing a matrix to an upper triangular matrix from via row operation, that is, Gaussian elimination.

The primary purpose of this paper is to provide a new totally positive matrix generated from a totally nonneg-

How to cite this paper: Ramadan, M.A. and Murad, M.M.A. (2015) Generating Totally Positive Toeplitz Matrix from an Upper Bidiagonal Matrix. Advances in Linear Algebra \& Matrix Theory, 5, 143-149. 
ative one and to construct its factorization.

The organization of our paper is as follows. In Section 2, we introduce our notation and give some auxiliary results which we use in the subsequent sections. In Section 3, we recall from [3] the Toeplitz matrices specified for the case $t_{k}=\left(\begin{array}{l}n \\ k\end{array}\right)$, on which our proofs heavily rely. In Section 4, we present the proofs of our main results. In last section, we present the factorization of this resulted matrix.

\section{Notation and Auxiliary Results}

\subsection{Notations}

In this subsection we introduce the notation that will be used in developing the paper. For $k, n$ we denote by $Q_{k, n}$ the set of all strictly increasing sequences of $k$ integers chosen from $\{1,2, \cdots, n\}$. For $\alpha=\left\{\alpha_{1}, \cdots, \alpha_{k}\right\}$, $\beta=\left\{\beta_{1}, \cdots, \beta_{k}\right\} \in Q_{k, n}$, we denote by $A[\alpha \mid \beta]$ the $k \times k$ submatrix of $A$ contained in the rows indexed by $\alpha_{1}, \cdots, \alpha_{k}$ and columns indexed by $\beta_{1}, \cdots, \beta_{k}$. A matrix $A$ is called totally positive (abbreviated TP henceforth) and totally nonnegative (abbreviated TN) if $\operatorname{det} A[\alpha \mid \beta]>0$ and $\operatorname{det} A[\alpha \mid \beta] \geq 0$, respectively, for all $\alpha$, $\beta \in Q_{k, n}, k=1,2, \cdots, n$. If a totally nonnegative matrix is also nonsingular, we write NsTN.

Definition 2.1.1 [3]

A square lower (upper) triangular matrix $A$ is called lower (upper) triangular positive matrix, denoted LTP (UTP), if for all $k=1, \cdots, n$ and for $\alpha=\left\{\alpha_{1}, \cdots, \alpha_{k}\right\}, \beta=\left\{\beta_{1}, \cdots, \beta_{k}\right\} \in Q_{k, n}$ with the property that $\alpha_{s} \geq \beta_{s}$ $\left(\beta_{s} \geq \alpha_{s}\right.$ ) for $s=1, \cdots, k$, then $\operatorname{det} A[\alpha \mid \beta]>0$.

Let $I$ be the square identity matrix of order $n$, and for $1 \leq i, j \leq n$, we let $E_{i j}$ be the square standard basis matrix whose only nonzero entry is 1 that occurs in the $(i, j)$ position.

A tridiagonal matrix that is also upper (lower) triangular is called an upper (lower) bidiagonal matrix. Statements referring to just triangular or bidiagonal matrices without the adjectives "upper" or "lower" may be applied to either case.

\subsection{Auxiliary Results}

We use the following classic formula known as Cauchy-Binet formula and stated in the theorem below.

Theorem 2.2.1 (Cauchy-Binet formula) ([4], p. 27). Let $A$ be an $n \times m$ matrix and $B$ be an $m \times p$ matrix then for each pair of indexed sets $\alpha \subseteq\{1, \cdots, n\}$ and $\beta \subseteq\{1, \cdots, p\}$ of cardinality $k$, where $1 \leq k \leq \min \{n, m$, $p\}$, we have

$$
\operatorname{det} A B[\alpha, \beta]=\sum_{\eta,|\eta|=k} \operatorname{det} A[\alpha, \eta] \cdot \operatorname{det} B[\eta, \beta]
$$

The following remarkable result is one of the most important and useful results in the study of TN matrices. This result first appeared in [5] see also [1] for another proof of this fact.

Theorem 2.2.2. Let $A=\left(a_{i j}\right)_{i, j=1}^{n}$ be a square matrix of order $n$. Then $A$ is NsTN if and only if $A$ has an $L U$ factorization, such that both $L$ and $U$ are NsTN square matrices.

Using this theorem and Cauchy-Binet formula we have the following corollary.

Corollary 2.2.3 [6]. Let $A=\left(a_{i j}\right)_{i, j=1}^{n}$ be a square matrix of order $n$. Then $A$ is TP if and only if $A$ has an $L U$ factorization, such that both $L$ and $U$ are TP square matrices.

We have the following theorem to prove both $L$ and $U$ are totally positive.

Theorem 2.2.4. Let $U=\left(u_{i j}\right)_{i, j=1}^{n}$ be an upper triangular square matrix of order $n$ satisfying

$U[1, \cdots, k \mid j+1, \cdots, j+k]>0$ for $j=0,1, \cdots, n-k, k=1, \cdots, n$

Then $U$ is UTP (upper totally positive). Similarly, if $L=\left(l_{i j}\right)_{i, j=1}^{n}$ is an lower triangular square matrix of order $n$ satisfying $L[i+1, \cdots, i+k \mid 1, \cdots, k]>0$ for $j=0,1, \cdots, n-k, k=1, \cdots, n$. Then $L$ is $L T P$ (lower totally positive). 
In the sequel we will make use the the following lemma, see, e.g. [7].

Lemma 2.2.5 (Sylvester Identity)

Partition square matrix $T$ of order $n, n>2$, as:

$$
T=\left[\begin{array}{ccc}
c_{1} & T_{12} & c_{2} \\
T_{21} & T_{22} & T_{23} \\
c_{3} & T_{32} & c_{4}
\end{array}\right]
$$

where $T_{22}$ square matrix of order $n-2$ and $c_{1}, c_{2}, c_{3}$ and $c_{4}$ are scalars. Define the submatrices

$$
T_{1}=\left[\begin{array}{cc}
c_{1} & T_{12} \\
T_{21} & T_{22}
\end{array}\right], T_{2}=\left[\begin{array}{cc}
T_{12} & c_{2} \\
T_{22} & T_{23}
\end{array}\right], T_{3}=\left[\begin{array}{cc}
T_{21} & T_{22} \\
c_{3} & T_{32}
\end{array}\right], T_{4}=\left[\begin{array}{ll}
T_{22} & T_{23} \\
T_{32} & c_{4}
\end{array}\right]
$$

Then if $T_{22}$ is non singular

$$
\operatorname{det} T=\frac{\operatorname{det} T_{1} \operatorname{det} T_{4}-\operatorname{det} T_{2} \operatorname{det} T_{3}}{\operatorname{det} T_{22}}
$$

\section{Toeplitz Matrices}

Assuming we are given a finite sequence $t_{-n}, t_{-n+1}, \cdots, t_{-1}, t_{0}, t_{1}, \cdots, t_{n}$ of distinct real numbers, the associated Toeplitz matrix is defined by $T=\left(t_{j-i}\right)_{i, j=1}^{n}$ or $T=\left(t_{i-j}\right)_{i, j=1}^{n}$. If we are given a one-sided finite sequence $t_{0}, t_{1}, \cdots, t_{n}$, then we understand this to mean that $t_{-k}=0, k=1,2, \cdots, n$ in the above definition. Sequences that give rise to totally positive Toeplitz matrices have been totally characterized in terms of their generating functions, i.e. representations of $\sum_{k=-n}^{n} t_{k} x^{k}$.

In our case, the normalization $t_{0}=1$, the sequence $t_{0}, t_{1}, \cdots, t_{n}$ gives rise to a totally positive Toeplitz matrix $T=\left(t_{j-i}\right)_{i, j=1}^{n}$ if and only if $\sum_{k=0}^{n} t_{k} x^{k}$ has the form

$$
\mathrm{e}^{\lambda x} \frac{\prod_{s=1}^{n}\left(1+i_{s} x\right)}{\prod_{s=0}^{n}\left(1-j_{s} x\right)}
$$

where $\lambda \geq 0, i_{s} \geq 0, j_{s} \geq 0$.

Now consider the polynomial $f(x)=(1+x)^{n}$, the upper triangular Toeplitz matrix

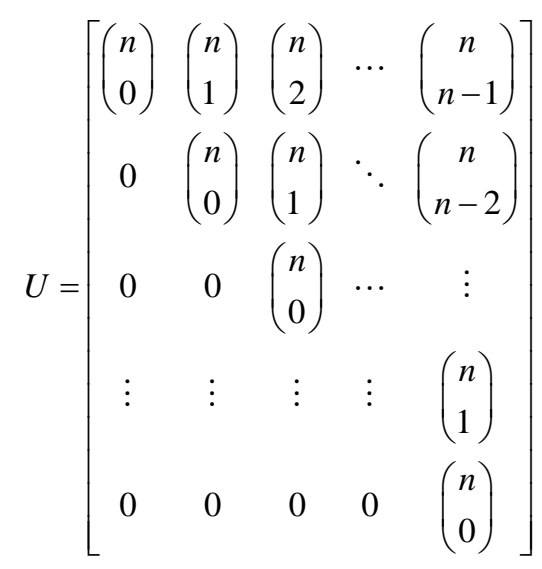

is $T P$. 


\section{Generating New Form of Toeplitz Matrix}

\subsection{Main Result}

Now we formalize the structure of our result by the following theorem.

Theorem 4.1.1. Assume that we are given the sequence $x_{1}, \cdots, x_{n-1}$ of $(n-1)$ distinct positive real numbers. Define the upper bidiagonal matrix $P=\left(p_{i j}\right)_{i, j=1}^{n}$ by

$$
p_{i j}= \begin{cases}x_{i} & \text { if } j=i+1 \\ 0 & \text { otherwise }\end{cases}
$$

That is the sequence $x_{1}, \cdots, x_{n-1}$ lies on the superdiagonal. Then the matrix $T$ defined as

$$
T=f(P)=\sum_{r=0}^{n} \sum_{k=0}^{n}\left(\begin{array}{l}
n \\
r
\end{array}\right)\left(\begin{array}{l}
n \\
k
\end{array}\right) P^{r}\left(P^{\mathrm{T}}\right)^{k}
$$

is $T P$.

\section{Proof}

To prove this result we must note that

$$
T=f(P)=\sum_{r=0}^{n} \sum_{k=0}^{n}\left(\begin{array}{l}
n \\
r
\end{array}\right)\left(\begin{array}{l}
n \\
k
\end{array}\right) P^{r}\left(P^{\mathrm{T}}\right)^{k}=\left(\sum_{r=0}^{n}\left(\begin{array}{l}
n \\
r
\end{array}\right) P^{r}\right) \times\left(\sum_{k=0}^{n}\left(\begin{array}{l}
n \\
k
\end{array}\right)\left(P^{\mathrm{T}}\right)^{k}\right)=U L
$$

where $U=\left(\sum_{r=0}^{n}\left(\begin{array}{l}n \\ r\end{array}\right) P^{r}\right)$ is upper triangular matrix and $L=\left(\sum_{k=0}^{n}\left(P^{\mathrm{T}}\right)^{k}\right)$ is lower triangular matrix. By corollary 2.2.3 $A$ is TP if both $U$ and $L$ are TP.

So, want to prove $U=\left(\sum_{r=0}^{n}\left(\begin{array}{l}n \\ r\end{array}\right) P^{r}\right)$ is upper TP.

$$
U=\left[\begin{array}{ccccc}
\left(\begin{array}{l}
n \\
0
\end{array}\right) & \left(\begin{array}{l}
n \\
1
\end{array}\right) x_{1} & \left(\begin{array}{l}
n \\
2
\end{array}\right) x_{1} x_{2} & \cdots & \left(\begin{array}{c}
n \\
n-1
\end{array}\right) \prod_{i=1}^{n-1} x_{i} \\
0 & \left(\begin{array}{l}
n \\
0
\end{array}\right) & \left(\begin{array}{l}
n \\
1
\end{array}\right) x_{2} & \ddots & \left(\begin{array}{c}
n \\
n-2
\end{array}\right) \prod_{i=1}^{n-2} x_{i} \\
0 & 0 & \left(\begin{array}{l}
n \\
0
\end{array}\right) & \ldots & \vdots \\
\vdots & \vdots & \vdots & \vdots & \left(\begin{array}{c}
n \\
1
\end{array}\right) x_{n-1} \\
0 & 0 & 0 & 0 & \left(\begin{array}{l}
n \\
0
\end{array}\right)
\end{array}\right]
$$

By Theorem 2.2.4 $U$ is $T P$ if

$$
U[1, \cdots, k \mid j+1, \cdots, j+k]=q\left|\begin{array}{cccc}
\left(\begin{array}{c}
n \\
j
\end{array}\right) & \left(\begin{array}{c}
n \\
j+1
\end{array}\right) & \cdots & \left(\begin{array}{c}
n \\
j+k-1
\end{array}\right) \\
\left(\begin{array}{c}
n \\
j-1
\end{array}\right) & \left(\begin{array}{c}
n \\
j
\end{array}\right) & \cdots & \left(\begin{array}{c}
n \\
j+k-2
\end{array}\right) \\
\vdots & \vdots & \ddots & \vdots \\
\left(\begin{array}{c}
n \\
j-k+1
\end{array}\right) & \left(\begin{array}{c}
n \\
j-k
\end{array}\right) & \cdots & \left(\begin{array}{c}
n \\
j
\end{array}\right)
\end{array}\right|>0
$$


where $q=\left(\prod_{k=1}^{\frac{n}{2}+1}\left(x_{k}\right)^{k}\right)\left(\prod_{k=1}^{\frac{n}{2}}\left(x_{n-k}\right)^{k}\right)$ which is positive and

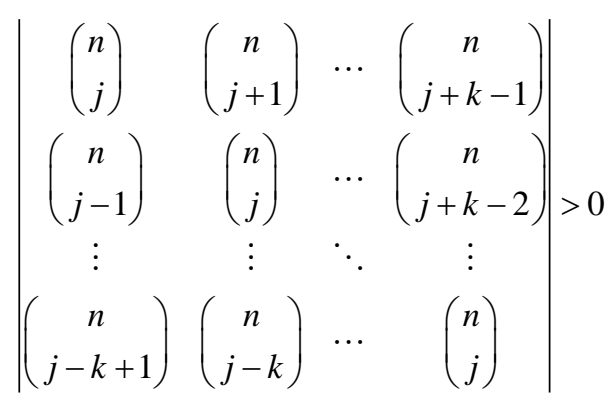

Since its submatrix of Toeplitz matrix.

\section{Illustrative Example}

Let we have the following sequence of distinct positive real numbers $1,4,3$.

Define the matrix $A$ as:

$$
A=\left[\begin{array}{llll}
0 & 1 & 0 & 0 \\
0 & 0 & 4 & 0 \\
0 & 0 & 0 & 3 \\
0 & 0 & 0 & 0
\end{array}\right]
$$

Then the matrix function

$$
\begin{aligned}
& f(A)=\left(\left(\begin{array}{l}
4 \\
0
\end{array}\right) A^{0}+\left(\begin{array}{l}
4 \\
1
\end{array}\right) A^{1}+\left(\begin{array}{l}
4 \\
2
\end{array}\right) A^{2}+\left(\begin{array}{l}
4 \\
3
\end{array}\right) A^{3}+\left(\begin{array}{l}
4 \\
4
\end{array}\right) A^{4}\right) \\
& \times\left(\left(\begin{array}{l}
4 \\
0
\end{array}\right)\left(A^{\mathrm{T}}\right)^{0}+\left(\begin{array}{l}
4 \\
1
\end{array}\right)\left(A^{\mathrm{T}}\right)^{1}+\left(\begin{array}{l}
4 \\
2
\end{array}\right)\left(A^{\mathrm{T}}\right)^{2}+\left(\begin{array}{l}
4 \\
3
\end{array}\right)\left(A^{\mathrm{T}}\right)^{3}+\left(\begin{array}{l}
4 \\
4
\end{array}\right)\left(A^{\mathrm{T}}\right)^{4}\right) \\
& =\left(I+4 A+6 A^{2}+4 A^{3}+A^{4}\right) \times\left(I+4 A^{\mathrm{T}}+6\left(A^{\mathrm{T}}\right)^{2}+4\left(A^{\mathrm{T}}\right)^{3}+\left(A^{\mathrm{T}}\right)^{4}\right) \\
& =\left(\left[\begin{array}{llll}
1 & 0 & 0 & 0 \\
0 & 1 & 0 & 0 \\
0 & 0 & 1 & 0 \\
0 & 0 & 0 & 1
\end{array}\right]+4 \times\left[\begin{array}{cccc}
0 & 1 & 0 & 0 \\
0 & 0 & 4 & 0 \\
0 & 0 & 0 & 3 \\
0 & 0 & 0 & 0
\end{array}\right]+6 \times\left[\begin{array}{cccc}
0 & 0 & 4 & 0 \\
0 & 0 & 0 & 12 \\
0 & 0 & 0 & 0 \\
0 & 0 & 0 & 0
\end{array}\right]+4 \times\left[\begin{array}{cccc}
0 & 0 & 0 & 12 \\
0 & 0 & 0 & 0 \\
0 & 0 & 0 & 0 \\
0 & 0 & 0 & 0
\end{array}\right]+\left[\begin{array}{cccc}
0 & 0 & 0 & 0 \\
0 & 0 & 0 & 0 \\
0 & 0 & 0 & 0 \\
0 & 0 & 0 & 0
\end{array}\right]\right) \\
& \times\left(\left[\begin{array}{llll}
1 & 0 & 0 & 0 \\
0 & 1 & 0 & 0 \\
0 & 0 & 1 & 0 \\
0 & 0 & 0 & 1
\end{array}\right]+4 \times\left[\begin{array}{cccc}
0 & 0 & 0 & 0 \\
1 & 0 & 0 & 0 \\
0 & 4 & 0 & 0 \\
0 & 0 & 3 & 0
\end{array}\right]+6 \times\left[\begin{array}{cccc}
0 & 0 & 0 & 0 \\
0 & 0 & 0 & 0 \\
4 & 0 & 0 & 0 \\
0 & 12 & 0 & 0
\end{array}\right]+4 \times\left[\begin{array}{cccc}
0 & 0 & 0 & 0 \\
0 & 0 & 0 & 0 \\
0 & 0 & 0 & 0 \\
12 & 0 & 0 & 0
\end{array}\right]+\left[\begin{array}{cccc}
0 & 0 & 0 & 0 \\
0 & 0 & 0 & 0 \\
0 & 0 & 0 & 0 \\
0 & 0 & 0 & 0
\end{array}\right]\right) \\
& =\left[\begin{array}{cccc}
1 & 4 & 24 & 48 \\
0 & 1 & 16 & 72 \\
0 & 0 & 1 & 12 \\
0 & 0 & 0 & 1
\end{array}\right] \times\left[\begin{array}{cccc}
1 & 0 & 0 & 0 \\
4 & 1 & 0 & 0 \\
24 & 16 & 1 & 0 \\
48 & 72 & 12 & 1
\end{array}\right]=\left[\begin{array}{cccc}
2897 & 3844 & 600 & 48 \\
3844 & 5441 & 880 & 72 \\
600 & 880 & 145 & 12 \\
48 & 72 & 12 & 1
\end{array}\right]
\end{aligned}
$$

is $T P$. 


\subsection{Properties}

1) Note that $\operatorname{det} T=1$ since $T=\left(\sum_{r=0}^{n}\left(\begin{array}{l}n \\ r\end{array}\right) P^{r}\right) \times\left(\sum_{k=0}^{n}\left(\begin{array}{l}n \\ k\end{array}\right)\left(P^{T}\right)^{k}\right)=U L$ and

$$
\operatorname{det} T=\operatorname{det} U L=\operatorname{det} U \operatorname{det} L=1 \times 1=1
$$

Using this property we prove the following lemma

Lemma 4.2.1. The matrix $T$, as defined above has the following property

$$
\operatorname{det} T_{22}=\operatorname{det} T_{1} \operatorname{det} T_{4}-\operatorname{det} T_{2} \operatorname{det} T_{3}
$$

where $T_{1}, T_{2}, T_{3}, T_{4}$ and $T_{22}$ are defined in Lemma 2.2.5.

\section{Proof}

The statement follows by Lemma 2.2.5 and the idea of $\operatorname{det} T=1$.

2) Let $P$ denote the square matrix of order n permutation matrix by the permutation

$p_{i j}=p_{n-i+1, n-j+1}, \quad 1 \leq i, j \leq n$, and suppose $T$ is a square $T P$ Toeplitz matrix. Then $P T P=L U$ is $T P$ too (see

[7]). Moreover, $S T^{-1} S$ is $T P$, where $S$ is diagonal matrix with diagonal entries alternately 1 and -1 .

3) The Hadamrd product of two TP toeplitz matrices is $T P$ matrix too, that is if we are given two square $T P$ matrices $T=\left[t_{i j}\right]$ and $W=\left[w_{i j}\right]$ of order $n$. Then the Hadamard product $Y=\left[y_{i j}\right]=\left[t_{i j} \cdot w_{i j}\right]$ is $T P$.

\section{Factorization}

\subsection{Construct New Factorization}

Our aim is to write the new TP Toeplitz matrix $T$ as a product of elementary matrices of a special form. For any $k \in\{2, \cdots, n\}$, we let $E_{k}^{r}(\delta)=\left[e_{i j}\right]$ to be the elementary lower matrix whose entries are defined by

$$
e_{i j}= \begin{cases}1 & \text { if } i=j \\ \delta & \text { if } i=k, j=k-r \\ 0 & \text { otherwise }\end{cases}
$$

Note that $E_{k}^{r}(\delta)$ can be written as $E_{k}^{r}(\delta)=I+\delta E_{k, k-r}$, where $I$ is square identity matrix of order $n$ and $E_{i, j}$ is square matrix of order $n$ whose non-zero entry is a 1 in the $(i, j)^{\text {th }}$ position $n$. Also, notice that $E_{k}^{r}(-\delta)=E_{k}^{r}(\delta)^{-1}$.

We use the elementary matrices $E_{k}^{r}(\delta)$ to reduce Lower diagonal matrix to identity matrix.

For example, we can consider the following $4 \times 4$ Lower diagonal matrix $L$

$$
L=\left[\begin{array}{llll}
1 & 0 & 0 & 0 \\
4 & 1 & 0 & 0 \\
6 & 4 & 1 & 0 \\
4 & 6 & 4 & 1
\end{array}\right]
$$

It can be factorized as

$$
\begin{aligned}
L & =E_{2}^{1}(4) E_{3}^{2}(6) E_{3}^{1}(4) E_{4}^{3}(4) E_{4}^{2}(6) E_{4}^{1}(4) I \\
& =\left(E_{2}^{1}(1)\right)^{4}\left(E_{3}^{2}(1)\right)^{6}\left(E_{3}^{1}(1)\right)^{4}\left(E_{4}^{3}(1)\right)^{4}\left(E_{4}^{2}(1)\right)^{6}\left(E_{4}^{1}(1)\right)^{4} I
\end{aligned}
$$

\subsection{General Characterization}

We begin a definition and a result that characterize the TP Toeplitz matrix $T$ in terms of the elementary matrices $E_{k}^{l}(1)$.

Theorem 5.2.1. Any square Toeplitz matrix of oreder $n, T=\left[t_{i j}\right]$ can be written as 


$$
\begin{aligned}
T & =U L=\left[\begin{array}{ccccc}
1 & u_{12} & u_{13} & \cdots & u_{1 n} \\
0 & 1 & u_{23} & \cdots & u_{2 n} \\
0 & 0 & 1 & \cdots & u_{3 n} \\
\vdots & \vdots & \vdots & \ddots & \vdots \\
0 & 0 & 0 & \cdots & 1
\end{array}\right]\left[\begin{array}{ccccc}
1 & 0 & 0 & \cdots & 0 \\
l_{21} & 1 & 0 & \cdots & 0 \\
l_{31} & l_{32} & 1 & \cdots & 0 \\
\vdots & \vdots & \vdots & \ddots & \vdots \\
l_{n 1} & l_{n 2} & l_{n 3} & \cdots & 1
\end{array}\right] \\
= & \left(\left(E_{n}^{1}(1)\right)^{u_{(n-1) n}}\right)^{\mathrm{T}}\left(\left(E_{n}^{2}(1)\right)^{u_{(n-2) n}}\right)^{\mathrm{T}} \cdots\left(\left(E_{4}^{3}(1)\right)^{u_{14}}\right)^{\mathrm{T}}\left(\left(E_{3}^{1}(1)\right)^{u_{23}}\right)^{\mathrm{T}}\left(\left(E_{3}^{2}(1)\right)^{u_{13}}\right)^{\mathrm{T}}\left(\left(E_{2}^{1}(1)\right)^{u_{12}}\right)^{\mathrm{T}} I \\
& \cdot\left(E_{2}^{1}(1)\right)^{l_{21}}\left(E_{3}^{2}(1)\right)^{l_{31}}\left(E_{3}^{1}(1)\right)^{l_{32}}\left(E_{4}^{3}(1)\right)^{l_{41}} \cdots\left(E_{n}^{2}(1)\right)^{l_{n(n-2)}}\left(E_{n}^{1}(1)\right)^{l_{n(n-1)}}
\end{aligned}
$$

That is, $T=\prod_{k=2}^{n} \prod_{i=2}^{k}\left(\left(E_{n-k+2}^{n-k+1}(1)\right)^{u_{n-k+1, n-k+2}}\right)^{\mathrm{T}} \times I \times \prod_{k=2}^{n} \prod_{i=2}^{k}\left(E_{k}^{k-i+1}(1)\right)^{l_{k, i-1}}$

\section{Illustrative Example}

Let

$$
T=\left[\begin{array}{cccc}
2897 & 3844 & 600 & 48 \\
3844 & 5441 & 880 & 72 \\
600 & 880 & 145 & 12 \\
48 & 72 & 12 & 1
\end{array}\right]=\left[\begin{array}{cccc}
1 & 4 & 24 & 48 \\
0 & 1 & 16 & 72 \\
0 & 0 & 1 & 12 \\
0 & 0 & 0 & 1
\end{array}\right] \times\left[\begin{array}{cccc}
1 & 0 & 0 & 0 \\
4 & 1 & 0 & 0 \\
24 & 16 & 1 & 0 \\
48 & 72 & 12 & 1
\end{array}\right]
$$

The matrix in this example can be factorized as

$$
\begin{aligned}
T= & \left(\left(E_{4}^{1}(1)\right)^{12}\right)^{\mathrm{T}} \times\left(\left(E_{4}^{2}(1)\right)^{72}\right)^{\mathrm{T}} \times\left(\left(E_{4}^{3}(1)\right)^{48}\right)^{\mathrm{T}} \times\left(\left(E_{3}^{1}(1)\right)^{16}\right)^{\mathrm{T}} \times\left(\left(E_{3}^{2}(1)\right)^{24}\right)^{\mathrm{T}} \times\left(\left(E_{2}^{1}(1)\right)^{4}\right)^{\mathrm{T}} \times I \\
& \times\left(E_{2}^{1}(1)\right)^{4} \times\left(E_{3}^{2}(1)\right)^{24} \times\left(E_{3}^{1}(1)\right)^{16} \times\left(E_{4}^{3}(1)\right)^{48} \times\left(E_{4}^{2}(1)\right)^{72} \times\left(E_{4}^{1}(1)\right)^{12}
\end{aligned}
$$

Note that the number of the factored matrices equal

$$
2 \times(12+72+48+16+24+4)=352 \text { matrix }
$$

\section{References}

[1] Ando, T. (1987) Totally Positive Matrices. Linear Algebra and its Applications, 90, 165-219. http://dx.doi.org/10.1016/0024-3795(87)90313-2

[2] Fallat, S.M. (2007) Totally Positive and Totally Nonnegative Matrices. Chapter 21 in Handbook of Linear Algebra, In: Hogben, L., Ed., Chapman \& Hall/CRC, Boca Raton.

[3] Pinkus, A. (2010) Totally Positive Matrices, Cambridge Tracts. Vol.181, Cambridge Univ. Press, Cambridge.

[4] Fallat, S.M. and Johnson, C.R. (2011) Totally Nonnegative Matrices, Princeton Ser. Appl. Math. Princeton University Press, Princeton, Oxford.

[5] Cryer, C.W. (1973) The LU-Factorization of Totally Positive Matrices. Linear Algebra and its Applications, 7, 83-92. http://dx.doi.org/10.1016/0024-3795(73)90039-6

[6] Fallat, S.M. (2001) Bidiagonal Factorizations of Totally Nonnegative Matrices. American Mathematical Monthly, 108, 697-712. http://dx.doi.org/10.2307/2695613

[7] Adm, M. and Garloff, J. (2013) Interval of Totally Nonnegative Matrices. Linear Algebra and its Applications, 439, 3796-3806. 